\title{
Validação de fluxo para integralidade do cuidado: implicações da Saúde Pública e
}

\section{Suplementar}

\author{
Validation of a flow for integrality of care: implications from Public and Supplemental Health \\ Validación de flujo para la atención integral: implicaciones de la Salud Pública y Complementaria
}

Recebido: 31/10/2021 | Revisado: 08/11/2021 | Aceito: 10/11/2021 | Publicado: 15/11/2021

\author{
Naara Regia Pinheiro Cavalcante \\ ORCID: https://orcid.org/0000-0003-0261-9168 \\ Universidade Estadual do Ceará, Brasil \\ E-mail: naarapinheiro@gmail.com \\ Ilse Maria Tigre de Arruda Leitão \\ ORCID: https://orcid.org/0000-0002-6138-5448 \\ Universidade Estadual do Ceará, Brasil \\ E-mail: ilsetigre@ hotmail.com \\ Dina Mara Formiga da Silva \\ ORCID: https://orcid.org/0000-0002-5387-7194 \\ Universidade Estadual do Ceará, Brasil \\ E-mail: dinamara_silva@hotmail.com \\ Maria Salete Bessa Jorge \\ ORCID: https://orcid.org/0000-0001-6461-3015 \\ Universidade Estadual do Ceará, Brasil \\ E-mail: maria.salete.jorge@gmail.com
}

\begin{abstract}
Resumo
Objetivo: validar um fluxograma para articulação da saúde pública e suplementar sob a ótica da integralidade do cuidado. Metodologia: O estudo faz parte da pesquisa de mestrado intitulada: "construção e validação de um fluxograma para articulação da saúde pública e suplementar sob a ótica da integralidade do cuidado". É uma investigação de natureza metodológica, que foi composto por: FASE I - realização de entrevistas semiestruturadas; FASE II -elaboração do fluxograma; e FASE III -Validação de Conteúdo e Aparência. Após codificação do material obtido nas entrevistas, foram realizadas análises de conteúdo categorial clássica e com suporte do NVivo, cujos dados foram utilizados para compor a primeira versão do fluxograma. A primeira versão da ferramenta passou pela validação de conteúdo e de aparência. Resultados e Discussão: O resultado das validações trouxe escores de $100 \%$ de concordância dos Juízes de Conteúdo $(I V C=1,00)$ e classificou o fluxograma como "superior" conforme medida do instrumento SAM. A partir do retorno das avaliações dos especialistas, os itens aceitos no fluxograma foram mantidos e os itens que tiveram apontamentos, foram ajustados, gerando a versão final do fluxograma. Conclusão: O fluxo proposto se constitui em proposta inovadora para o Sistema de Saúde e pretende contribuir para a sistematização de rotina para articulação da saúde pública e suplementar, considerando que atualmente os setores se articulam prioritariamente na perspectiva de ressarcimento ao SUS.
\end{abstract}

Palavras-chave: Assistência integral à saúde; Gestão em saúde; Saúde pública; Saúde suplementar.

\begin{abstract}
Objective: to validate a flowchart to articulate public health and supplement from the perspective of comprehensive care. Methodology: The study is part of the master's research entitled: "construction and validation of a flowchart for articulation of public and supplementary health from the perspective of comprehensive care". It is an investigation of a methodological nature, which was composed of: PHASE I - semi-structured interviews; PHASE II -elaboration of the flowchart; and PHASE III - Content and Appearance Validation. After coding the material obtained in the interviews, analyses of classical and nvivo-supported category content were performed, whose data were used to make up the first version of the flowchart. The first version of the tool went through content and appearance validation. Results and Discussion: The results of the validations brought scores of $100 \%$ agreement of the Content Judges (CVI $=1.00$ ) and classified the flowchart as "superior" according to the measure of the SAM instrument. From the return of the experts' evaluations, the items accepted in the flowchart were maintained and the items that had notes were adjusted, generating the final version of the flowchart. Conclusion: The proposed flow is an innovative proposal for the Health System and intends to contribute to the routine systematization for the articulation of public and supplementary health, considering that currently the sectors are articulated primarily in the perspective of reimbursement to the SUS.
\end{abstract}

Keywords: Comprehensive health care; Health management; Public health; Supplementary health. 


\begin{abstract}
Resumen
Objetivo: validar un diagrama de flujo para articular la salud pública y complementar desde la perspectiva de la atención integral. Metodología: El estudio forma parte de la investigación del máster titulada: "construcción y validación de un diagrama de flujo para la articulación de la salud pública y complementaria desde la perspectiva de la atención integral". Es una investigación de carácter metodológico, que estuvo compuesta por: FASE I - entrevistas semiestructuradas; FASE II - elaboración del diagrama de flujo; y FASE III - Validación de contenido y apariencia. Tras codificar el material obtenido en las entrevistas, se realizaron análisis de contenido de categorías clásicas y soportadas por nvivo, cuyos datos se utilizaron para componer la primera versión del diagrama de flujo. La primera versión de la herramienta pasó por la validación de contenido y apariencia. Resultados y Discusión: Los resultados de las validaciones trajeron puntajes de $100 \%$ de acuerdo de los Jueces de Contenido $(\mathrm{CVI}=1.00)$ y clasificaron el diagrama de flujo como "superior" según la medida del instrumento SAM. A partir de la devolución de las evaluaciones de los expertos, se mantuvieron los ítems aceptados en el diagrama de flujo y se ajustaron los ítems que tenían notas, generando la versión final del diagrama de flujo. Conclusión: El flujo propuesto es una propuesta innovadora para el Sistema de Salud y pretende contribuir a la sistematización rutinaria para la articulación de la salud pública y complementaria, considerando que actualmente los sectores se articulan principalmente en la perspectiva del reembolso al SUS.
\end{abstract}

Palabras clave: Atención integral de la salud; Gestión de la salud; Salud pública; Salud suplementaria.

\title{
1. Introdução
}

A Constituição Federal de 1988 garantiu a Saúde como direito de todos e dever do Estado, mediante políticas sociais e econômicas que visassem à redução do risco de doença e de outros agravos e ao acesso universal e igualitário às ações e serviços para sua promoção, proteção e recuperação, definiu o Sistema Único de Saúde (SUS) como uma rede regionalizada e hierarquizada constituída por ações e serviços públicos de saúde, organizados de acordo com diretrizes de descentralização, atendimento integral e participação da comunidade, bem como permitiu a participação da saúde suplementar no Sistema de Saúde Brasileiro, colocando que "a assistência à saúde é livre à iniciativa privada” (Ministério da Saúde, 1988a).

Nos termos da Constituição, esta configuração possibilitou a inserção de serviços privados no SUS, sendo estes denominados como complementares, e os não inseridos/vinculados ao SUS, como suplementares.

Para minimizar os efeitos da convivência paralela dos Setores público e privado, os sistemas têm passado por implementações em suas organizações ao longo dos últimos anos, e como um marco importante destaca-se a Saúde Suplementar que passou a ser regida pela Lei n. 9.656/98 e regulamentada no ano 2000, através da criação da Agência Nacional de Saúde Suplementar - ANS (Ministério da Saúde, 1998b)

A partir da regulamentação instituída, o Setor Suplementar, como parte integrante do Sistema de Saúde Brasileiro, passou a ter que atender exigências para a implantação de ações de saúde convergentes com o preconizado no Direito à Saúde.

A criação da ANS caracterizou-se pela unificação da regulação e da fiscalização numa agência reguladora única, vinculada ao Ministério da Saúde por um Contrato de Gestão. Esse processo caracterizou-se pela introdução de uma cultura regulatória não centrada somente no saneamento econômico e seus aspectos gerenciais, mas na busca de uma expressiva mudança da atenção à saúde, referendada pelo fato de essa regulação se dar no âmbito do Ministério da Saúde (Ministério da saúde, 2009).

Sendo a atenção integral eixo fundamental para o cuidado a saúde, e considerando o envelhecimento da população, a prevalência das doenças crônicas, a incorporação de novas tecnologias e ainda com vistas a garantir a própria sustentabilidade, a Saúde Suplementar precisou se reorganizar de modo a garantir que suas ações fossem convergentes com essa modalidade de assistência.

Dentro dessa perspectiva, a ANS, com objetivo de alcançar a integralidade da assistência e do cuidado, tem conduzido sua atuação para que as operadoras de planos de Saúde (OPS) sejam produtoras de saúde, e que trabalhem em prol da promoção da saúde e prevenção das doenças de seus beneficiários: 
A ANS tem buscado estimular as operadoras de planos de saúde a repensarem a organização do sistema de saúde com vistas a contribuir para mudanças que possibilitem sair do modelo hegemonicamente centrado na doença, em procedimentos e baseado na demanda espontânea, para um Modelo de Atenção Integral à Saúde, no qual haja incorporação progressiva de ações de Promoção da Saúde e Prevenção de Riscos e Doenças (Ministério da Saúde, 2011).

Embora, na Saúde Suplementar, sejam constatadas várias iniciativas relacionadas à implantação de ações de Promoção da Saúde e Prevenção de riscos e doenças, e que suas Resoluções Normativas estão fundamentadas nos princípios de intersetorialidade, integralidade, mobilização e controle social, informação, sustentabilidade, observa-se que os setores suplementar e público da saúde tem se articulado prioritariamente no que se refere ao ressarcimento ao SUS, que ocorre em razão dos atendimentos realizados na rede pública de saúde pelos beneficiários das operadoras, desde que esses serviços estejam também previstos nos contratos.

No contexto atual, os beneficiários dos planos de saúde, ficam anônimos para suas Operadoras, sendo identificados nesse segmento apenas por ocasião da apresentação de contas para o ressarcimento ao SUS. No que se refere a beneficiários de planos, em internamento no SUS, não se observa interface Público-privada, nem mecanismos de articulação entre estes setores, que permitam uma comunicação precoce entre eles, de modo que a integralidade do cuidado seja uma responsabilidade compartilhada.

Considerando que o Sistema de Saúde necessita de ações integradas que possibilitem um incremento positivo nas ações de saúde da população, este estudo tem como objetivo validar um fluxograma para articulação da saúde pública e suplementar sob a ótica da integralidade do cuidado.

\section{Metodologia}

O estudo faz parte da pesquisa de mestrado intitulada: “construção e validação de um fluxograma para articulação da saúde pública e suplementar sob a ótica da integralidade do cuidado”. É uma investigação de natureza metodológica, realizado no período de setembro a novembro/2018, no Município de Fortaleza-CE, junto à Coordenadoria de Regulação, Controle, Avaliação e Auditoria do Estado do Ceará (CORAC) e o Núcleo da ANS Fortaleza/CE. Foi desenvolveu por meio das seguintes etapas: FASE I - realização de entrevistas semiestruturada; FASE II - elaboração do fluxograma; e FASE III Validação de Conteúdo e Aparência (Joaquim \& Silvino, 2020).

Fizeram parte da população do estudo técnicos da CORAC e técnicos da ANS selecionados conforme os seguintes critérios de inclusão: assumir a função de Gestor, Assessor, Gerente, Supervisor, coordenador ou outros cargos ligados à chefia. A partir dos critérios estabelecidos foram selecionados 05 (cinco) técnicos que consentiram sua participação por meio de assinatura de Termo de Consentimento Livre e Esclarecido. A entrada no campo se deu inicialmente com a busca em fontes de dados secundários (literatura) e visitas a dependências governamentais supracitadas para realização das entrevistas.

$\mathrm{Na}$ fase I foi realizada entrevista semiestruturada com os participantes do estudo para a obtenção de informações referentes aos fluxos, estratégias e/ou processos existentes, assim como as propostas deles para identificação e sinalização dos cidadãos com planos de saúde em internação no SUS, para continuidade da integralidade do cuidado no setor suplementar. As entrevistas foram gravadas, após o consentimento dos participantes e transcritas na íntegra pela pesquisadora.

Com o conteúdo das entrevistas foi realizada uma análise de conteúdo categorial com suporte do NVivo, software que suporta métodos qualitativos e variados de pesquisa, organizando, analisando e encontrando informações em dados não estruturados ou qualitativos (Bringer et al., 2006). 
A partir do desenvolvimento das relações entre "Nós" e "Atributos", estruturados no software, a disposição dos dados na tela e a preparação de gráficos e diagramas foram ferramentas fundamentais que facilitaram a visualização do todo e a análise dos dados (Nascimento et al., 2018).

$\mathrm{Na}$ fase II do estudo, após a identificação dos itens primordiais para composição do fluxograma descritor, deu-se início a elaboração da ferramenta. De acordo com Franco (2003), o fluxograma descritor é uma representação gráfica do processo de trabalho, que permite um olhar agudo sobre os fluxos existentes na produção da assistência, possibilita detectar problemas, revelar os processos de trabalho que são produzidos, contribuindo para o planejamento e reorganização dos processos de trabalho.

A partir de cada entrevista realizada, foram esboçados 05 (cinco) fluxos individuais que foram associados para a construção da proposta do Fluxograma. Cada fluxo foi elaborado como uma representação gráfica da cadeia do processo de trabalho proposto para atender o objetivo deste estudo, na forma de um diagrama constituído por simbologias convencionadas universalmente, quais sejam: a elipse, representando sempre a entrada ou saída do processo de produção de serviços; o losango, indicando os momentos em que deve haver uma decisão para a continuidade do trabalho e o retângulo, que diz respeito ao momento de intervenção, ação, sobre o processo (Franco \& Merhy, 2003).

Para a análise dos dados desta fase, foi utilizada a análise de conteúdo categorial clássica. A análise de conteúdo refere-se a um conjunto de técnicas de análise com as quais se busca compreender as comunicações para além dos seus significados imediatos. A sutileza dos seus métodos de análise objetiva a superação de incertezas e o enriquecimento da leitura, ou seja, o desejo de rigor e a necessidade de descobrir e ir além das aparências (Bardin, 2011).

A proposta consolidada do Fluxograma Descritor, construída a partir das contribuições dos técnicos, foi submetida à transcrição por um Designer gráfico e se constituiu como $1^{\mathrm{a}}$ versão da ferramenta a ser complementada e aprimorada a partir da validação.

Na fase III foram realizadas as etapas de Validação de Conteúdo e Aparência. A validade do conteúdo de um instrumento baseia-se em um julgamento, e indica em que medida ele possui uma amostra apropriada de itens para medir o construto específico e medir de forma adequada o seu domínio. Para essa validade não há métodos totalmente objetivos (Polit $\&$ Beck, 2011).

Sobre a aparência, Abbagnano (1998) coloca que ela demonstra a realidade dos objetos como se apresentam às pessoas que irão realizar o julgamento, estando relacionada ao que se revela como essência dos objetos.

A Validade de Conteúdo foi realizada mediante consulta a 07 (sete) especialistas da área, distribuídos em dois grupos distintos: 03 juízes de conteúdo (pesquisadores/docentes na área de saúde Coletiva, Gestão em Saúde e/ou Validação de instrumentos) e 04 juízes técnicos (profissionais com experiência na área objeto do estudo). A Validade de Aparência foi realizada pelos mesmos juízes de conteúdo.

Para a seleção dos juízes, Lynn (1986) recomenda um mínimo de cinco e o máximo de dez e Vianna (1982) sugere que o número de especialistas seja ímpar para evitar o empate de opiniões.

A escolha dos juízes pesquisadores/docentes foi feita por amostragem intencional, na qual "os pesquisadores podem decidir propositalmente sobre a seleção de sujeitos considerados típicos da população ou conhecedores das questões estudadas (Fehring, 1994).

Para aprimorar a seleção da amostragem intencional, foram utilizados parâmetros para a seleção dos juízes por meio do sistema de classificação de juízes adaptado do sistema de pontuação de Fehring (1994). Na adaptação do sistema, os critérios para os Juízes de Conteúdo e de aparência (pesquisadores/ Docentes), foram: Doutor na área de interesse (2 pontos); Mestre na área de interesse (2 Pontos); Participação em bancas avaliadoras de Tese, Dissertação ou Monografia de graduação ou Especialização que envolva a temática na área de interesse (1 ponto); Experiência docente em Disciplina na área de 
interesse ( 2 pontos); Ter orientado Tese, Dissertações ou Monografias na área de interesse (2 pontos); Ter atuação prática na área de interesse (4 pontos ) e Experiência na temática de validação de instrumentos (1 ponto).

O total máximo de pontos foi 14, e as áreas de interesse consideradas foram: Saúde Coletiva; Gestão em saúde; Regulação em saúde e Saúde suplementar. Os pesquisadores/docentes com pontuação igual ou superior a cinco, foram convidados a participar da validação do estudo.

Os juízes técnicos também foram selecionados por amostragem intencional, acrescida dos critérios adaptados de Fehring (1994), em mesma metodologia aplicada para os Juízes pesquisadores/Docentes.

$\mathrm{Na}$ adaptação do sistema para este grupo de especialista (Juízes Técnicos), os critérios para os, foram: Doutorado na área de interesse (1 ponto); Mestrado na área de interesse (1 ponto); Especialização na área de interesse (1 ponto); Apresentação de trabalhos em eventos na área de interesse (1 ponto); Experiência docente na área de interesse (1 ponto); Experiência profissional em Gestão em Saúde (4 pontos); e Experiência profissional na área de Saúde Suplementar (5 pontos).

O total máximo de pontos foi 14, e as áreas de interesse consideradas foram: Gestão em saúde; Regulação em saúde, Saúde Suplementar. Os juízes técnicos com pontuação igual ou superior a 5, foram convidados a participar da validação do estudo.

Ressalta-se que dois respondentes da entrevista que se enquadraram nos critérios adaptados para os juízes técnicos, e por terem demonstrado disponibilidade e interesse na construção do fluxograma, foram considerados como participantes desta etapa de validação.

$\mathrm{Na}$ validação do respondente, o relato do investigador é comparado com os relatos dos investigados, para que seja estabelecido o nível de correspondência entre os dois grupos, sendo uma checagem forte para a credibilidade de um projeto de pesquisa (Pope \& Mays, 2009; Portugal, et al., 2021).

Aos Juízes que consentiram participar da validação, foi entregue pessoalmente a carta convite, Termo de Consentimento Livre e Esclarecido, os questionários de identificação dos avaliadores, a versão do fluxograma em PDF e os instrumentos de avaliação (Questionário de avaliação para os juízes pesquisadores/docentes e os juízes técnicos, e o Suitability Assesment of Materials (SAM) apenas para os juízes pesquisadores/docentes.

Foi solicitado que os juízes realizassem consulta ao fluxograma concomitante ao preenchimento do questionário de avaliação adaptado de Mesquita (2016), e baseado no modelo de Likert (1932), na qual deveriam assinalar sua concordância através da escala ordinal que considerou os seguintes itens ( 1 - discordo totalmente; 2 - discordo parcialmente; 3 - concordo parcialmente; 4 - concordo totalmente).

No instrumento foi fornecido um espaço para justificativas referentes aos itens elencados e para sugestões que os especialistas considerassem necessárias.

Para validação do conteúdo do Fluxograma, foi empregado o Índice de Validade de Conteúdo (IVC). Sobre o valor do IVC, o item é tido como representante para o elenco se houver concordância de cerca de $80 \%$ entre os juízes (Pasquali, 2013).

O IVC indica o grau em que o instrumento é válido em relação ao conteúdo. Baseia-se em classificações agregadas de um conjunto de especialistas, verificando em que medida as opiniões dos especialistas são congruentes (Polit \& Beck, 2011).

O escore do índice foi calculado por meio das somas de concordância dos itens marcados em 3 ou 4 pelos especialistas na escala tipo Likert. A fórmula para o cálculo do IVC é: $\mathrm{N}^{\circ}$ e respostas 3 ou $4 / \mathrm{N}^{\circ}$ total de respostas.

Para a validação de aparência foi utilizado o SAM, instrumento americano proposto por Doak, Doak e Root (Doak et al., 1996), traduzido e adequado do original em inglês para a língua portuguesa por Souza, Turrini e Poveda (2015). Sua aplicação é simples e o cálculo do escore total de adequação é feito a partir da soma dos escores obtidos, dividido pelo total de 
escores e multiplicado por 100 para transformar em percentual e o resultado dos pontos atribuídos a cada item categoriza o material em ‘superior' (70-100\%), ‘adequado' (40-69\%) e 'não adequado' (0-39\%) (Corrêa et al., 2021).

A realização do estudo foi aprovada pelo Comitê de Ética da Universidade Estadual do Ceará no Parecer Consubstanciado $\mathrm{N}^{\mathrm{o}} 2.872 .412$.

\section{Resultados e Discussão}

FASE I - as informações obtidas nas entrevistas e analisadas com suporte no Nvivo revelaram a inexistência de fluxos, estratégias e/ou processos atuais para identificação e sinalização de cidadãos com planos de saúde em internação do SUS para continuidade da integralidade do cuidado na Saúde Suplementar e que os Sistemas atualmente trabalham na perspectiva do ressarcimento ao SUS.

As propostas apresentadas para implementação de novo fluxo apontaram etapas sucintas com destaque a necessidade de conhecer melhor o sistema de informação, pois este ainda é restrito à prestação de assistência no âmbito hospitalar, sendo o principal elo entre o sistema público e privado, o financiamento (ressarcimento ao SUS). Os participantes sinalizaram a necessidade da identificação do paciente de uma forma integrada nos sistemas, tanto público como privado, com possibilidade de permuta de dados e informações para uma melhoria na prestação do cuidado de forma compartilhada / conjunta.

FASE II - A construção do fluxograma foi fundamentada metodologicamente nas orientações para a construção de diagramas. O trabalho inicial dessa fase se deu com a leitura das entrevistas transcritas e, com base no conteúdo das informações, foram destacadas as dimensões que representavam as etapas do fluxo proposto por cada indivíduo. Seguindo a sequência lógica das etapas de cada fluxo individual, itens correspondentes foram agrupados e associados dando a origem à proposta do Fluxograma Geral em sua $1^{\mathrm{a}}$ versão (Figura 1). 
Figura 1 - Fluxograma Geral ( $1^{\circ}$ Versão).

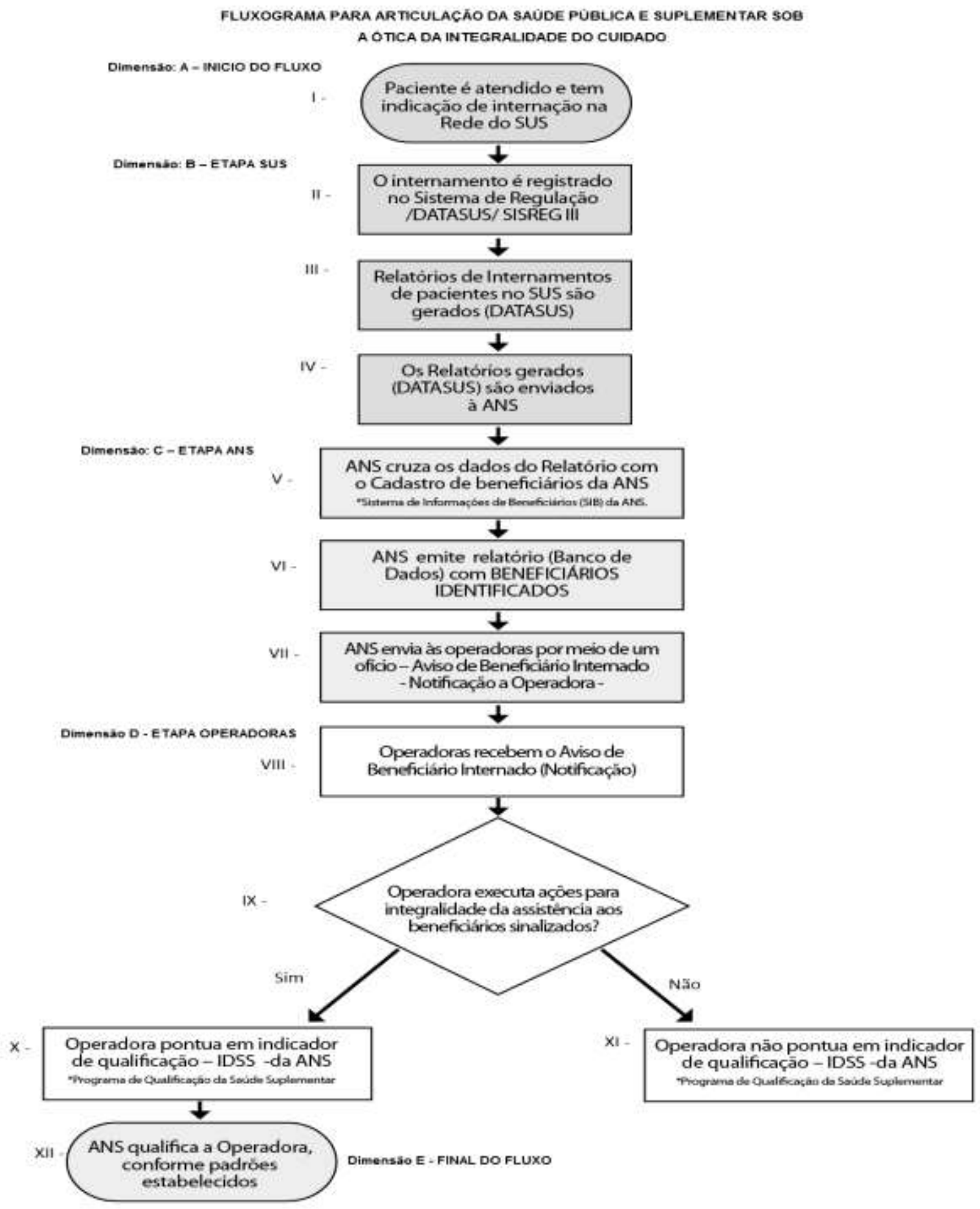

Fonte: Autores.

A $1^{\circ}$ versão do Fluxograma foi redesenhada por Designer Gráfico, em programa Corel Draw 8. Ao concluir a diagramação do Fluxograma, o design gráfico enviou a versão pré-validação, que foi impressa e enviada aos juízes especialistas com vistas à validação do conteúdo e aparência. 
FASE III - Após construção do Fluxograma, foi iniciada a etapa de validação de conteúdo e aparência. Para Validação do Conteúdo, as avaliações dos 07 (sete) Juízes Especialistas foram analisadas conforme as dimensões e etapas apresentadas no Fluxograma, sendo calculados os IVCs de cada etapa.

Como exposto no Quadro 1, a distribuição dos Índices de Validade de Conteúdo de acordo com as análises dos Especialistas (pesquisadores/docentes), teve escores de $100 \%$ de concordância dos Juízes (IVC = 1,00) em todas as etapas avaliadas. Nesta validação, apenas os itens I, III, IV e VII receberam escore 3 (concordo parcialmente) de um dos juízes, o que não afeta a validade da concordância, considerando o cálculo do IVC.

Os itens citados, pontuados com escore 3, foram apontados pelo Juiz no campo "parecer” como dúvida operacional do processo, não havendo a necessidade de serem adicionadas no escopo do fluxograma.

Quadro 1 - Distribuição dos Índices de Validade de Conteúdo, de acordo com análise dos Especialistas (pesquisadores/ docentes).

\begin{tabular}{|l|c|}
\hline ITENS & IVC \\
\hline GERAL & IVC \\
\hline Cor dos símbolos e fonte & 1,00 \\
\hline Estrutura/aspecto & 1,00 \\
\hline Dimensão: A - INICIO DO FLUXO & IVC \\
\hline I - Paciente é atendido e tem indicação de internação na Rede do SUS & 1,00 \\
\hline Dimensão: B - ETAPA SUS & IVC \\
\hline II - O internamento é registrado no Sistema de Regulação /DATASUS/ SISREG III & 1,00 \\
\hline III - Relatórios de Internamentos de pacientes no SUS são gerados (DATASUS) & 1,00 \\
\hline IV - Os Relatórios gerados (DATASUS) são enviados à ANS & 1,00 \\
\hline Dimensão: C - ETAPA ANS & IVC \\
\hline $\begin{array}{l}\text { V - ANS cruza os dados do Relatório com o Cadastro de beneficiários da ANS } \\
\text { *Sistema de Informações de Beneficiários (SIB) da ANS. }\end{array}$ & 1,00 \\
\hline VI - ANS emite relatório (Banco de Dados) com BENEFICIÁRIOS IDENTIFICADOS & 1,00 \\
\hline $\begin{array}{l}\text { VII - ANS envia às operadoras por meio de um ofício - Aviso de Beneficiário Internado - Notificação } \\
\text { a Operadora - }\end{array}$ & 1,00 \\
\hline Dimensão D - ETAPA OPERADORAS & IVC \\
\hline VIII - Operadoras recebem o Aviso de Beneficiário Internado (Notificação) & 1,00 \\
\hline IX - Operadora executa ações para integralidade da assistência aos beneficiários sinalizados? & 1,00 \\
\hline $\begin{array}{l}\text { X - SIM - Operadora pontua em indicador de qualificação - IDSS - da ANS } \\
\text { *Programa de Qualificação da Saúde Suplementar }\end{array}$ & 1,00 \\
\hline $\begin{array}{l}\text { XI - NÃO - Operadora não pontua em indicador de qualificação - IDSS -da ANS } \\
\text { *Programa de Qualificação da Saúde Suplementar }\end{array}$ & 1,00 \\
\hline Dimensão E - FINAL DO FLUXO & IVC \\
\hline XII - ANS qualifica a Operadora, conforme padrões estabelecidos & 1,00 \\
\hline
\end{tabular}

Fonte: Autores.

O Quadro 2, que traz a distribuição das análises dos Especialistas (Técnicos), mostra também escores de $100 \%$ de concordância $(\mathrm{IVC}=1,00)$ em todas as etapas avaliadas. Nesta validação, apenas os itens "Estrutura/aspecto", IX e X, receberam escore 3 (concordo parcialmente) em quantidade 01 cada, o que não afeta a validade da concordância.

O Juiz que pontuou o escore 3 nos itens IX e X apresentou contribuição para as etapas, sugerindo indicadores diferenciados a partir da atuação das operadoras. Por sua relevância, a sugestão foi incluída na versão final do Fluxograma. 
Quadro 2 - Distribuição dos Índices de Validade de Conteúdo, de acordo com análise dos Especialistas (Técnicos).

\begin{tabular}{|l|c|}
\hline ITENS & IVC \\
\hline GERAL & IVC \\
\hline Cor dos símbolos e fonte & 1,00 \\
\hline Estrutura/aspecto & 1,00 \\
\hline Dimensão: A - INICIO DO FLUXO & IVC \\
\hline I - Paciente é atendido e tem indicação de internação na Rede do SUS & 1,00 \\
\hline Dimensão: B - ETAPA SUS & IVC \\
\hline II - O internamento é registrado no Sistema de Regulação /DATASUS/ SISREG III & 1,00 \\
\hline III - Relatórios de Internamentos de pacientes no SUS são gerados (DATASUS) & 1,00 \\
\hline IV - Os Relatórios gerados (DATASUS) são enviados à ANS & 1,00 \\
\hline Dimensão: C - ETAPA ANS & IVC \\
\hline $\begin{array}{l}\text { V - ANS cruza os dados do Relatório com o Cadastro de beneficiários da ANS } \\
\text { *Sistema de Informações de Beneficiários (SIB) da ANS. }\end{array}$ & 1,00 \\
\hline VI - ANS emite relatório (Banco de Dados) com BENEFICIÁRIOS IDENTIFICADOS & 1,00 \\
\hline VII - ANS envia às operadoras por meio de um ofício - Aviso de Beneficiário Internado \\
- Notificação a Operadora - & IVC \\
\hline Dimensão D - ETAPA OPERADORAS & 1,00 \\
\hline VIII - Operadoras recebem o Aviso de Beneficiário Internado (Notificação) & 1,00 \\
\hline IX - Operadora executa ações para integralidade da assistência aos beneficiários sinalizados? & 1,00 \\
\hline $\begin{array}{l}\text { X - SIM - Operadora pontua em indicador de qualificação - IDSS - da ANS } \\
\text { *Programa de Qualificação da Saúde Suplementar }\end{array}$ & 1,00 \\
\hline $\begin{array}{l}\text { XI - NÃO - Operadora não pontua em indicador de qualificação - IDSS -da ANS } \\
\text { *Programa de Qualificação da Saúde Suplementar }\end{array}$ & IVC \\
\hline Dimensão E - FINAL DO FLUXO & 1,00 \\
\hline XII - ANS qualifica a Operadora, conforme padrões estabelecidos & \\
\hline
\end{tabular}

Fonte: Autores.

$\mathrm{Na}$ validação de aparência, realizada pelos Juízes Especialistas (Pesquisadores/Docentes) através do instrumento SAM, os percentuais de concordância classificaram o produto como "superior", habilitando-o quanto ao quesito avaliado. As porcentagens de concordância de cada ponto/ fator abordado no SAM encontram-se no Quadro 3. 
Quadro 3 - Grau de Concordância dos especialistas (Pesquisadores/ Docentes) - SAM.

\begin{tabular}{|c|c|c|c|}
\hline \multicolumn{4}{|l|}{1 - Conteúdo } \\
\hline Fator & Avaliador 1 & Avaliador 2 & Avaliador 3 \\
\hline O propósito está evidente & 2 & 2 & 2 \\
\hline O conteúdo está focado no propósito & 2 & 2 & 2 \\
\hline O conteúdo destaca os pontos principais & 2 & 2 & 2 \\
\hline \multicolumn{4}{|l|}{2 - Exigência de alfabetização } \\
\hline Fator & Avaliador 1 & Avaliador 2 & Avaliador 3 \\
\hline Nível de leitura & 2 & 2 & 2 \\
\hline Usa vocabulário com palavras comuns no texto & 1 & 2 & 2 \\
\hline O contexto vem antes de novas informações & 2 & 2 & 2 \\
\hline O aprendizado é facilitado por tópicos & 2 & 2 & 2 \\
\hline \multicolumn{4}{|l|}{3 - Ilustrações } \\
\hline Fator & Avaliador 1 & Avaliador 2 & Avaliador 3 \\
\hline O propósito da ilustração referente ao texto está claro & 2 & 2 & 2 \\
\hline \multicolumn{4}{|l|}{ 4-Leiaute e apresentação } \\
\hline Fator & Avaliador 1 & Avaliador 2 & Avaliador 3 \\
\hline Característica do leiaute & 2 & 2 & 2 \\
\hline Tamanho e tipo de letra & 1 & 2 & 2 \\
\hline \multicolumn{4}{|l|}{5 - Estimulação / Motivação do aprendizado } \\
\hline Fator & Avaliador 1 & Avaliador 2 & Avaliador 3 \\
\hline Utiliza a interação & 2 & 2 & 2 \\
\hline As orientações são específicas & 2 & 2 & 2 \\
\hline \multicolumn{4}{|l|}{ 6 - Adequação cultural } \\
\hline Fator & Avaliador 1 & Avaliador 2 & Avaliador 3 \\
\hline É semelhante a sua lógica, linguagem e experiência & 2 & 2 & 2 \\
\hline Imagem cultural & 2 & 2 & 2 \\
\hline \multirow[b]{2}{*}{ \% de CONCORDÂNCIA SAM } & Avaliador 1 & Avaliador 2 & Avaliador 3 \\
\hline & $\mathbf{9 2 , 8 \%}$ & $100 \%$ & $100 \%$ \\
\hline
\end{tabular}

Fonte: Autores.

A partir do retorno das avaliações dos especialistas pesquisadores/docentes e técnicos, os itens aceitos no fluxograma, conforme os consensos das respostas ao nível de concordância foram mantidos. Os itens IX e X que tiveram apontamentos, sugestões/recomendações, foram ajustados, gerando a versão final do fluxograma, apresentada na Figura 2. 
Figura 2 - Fluxograma para articulação da saúde pública e suplementar sob a ótica da integralidade do cuidado - Versão Final

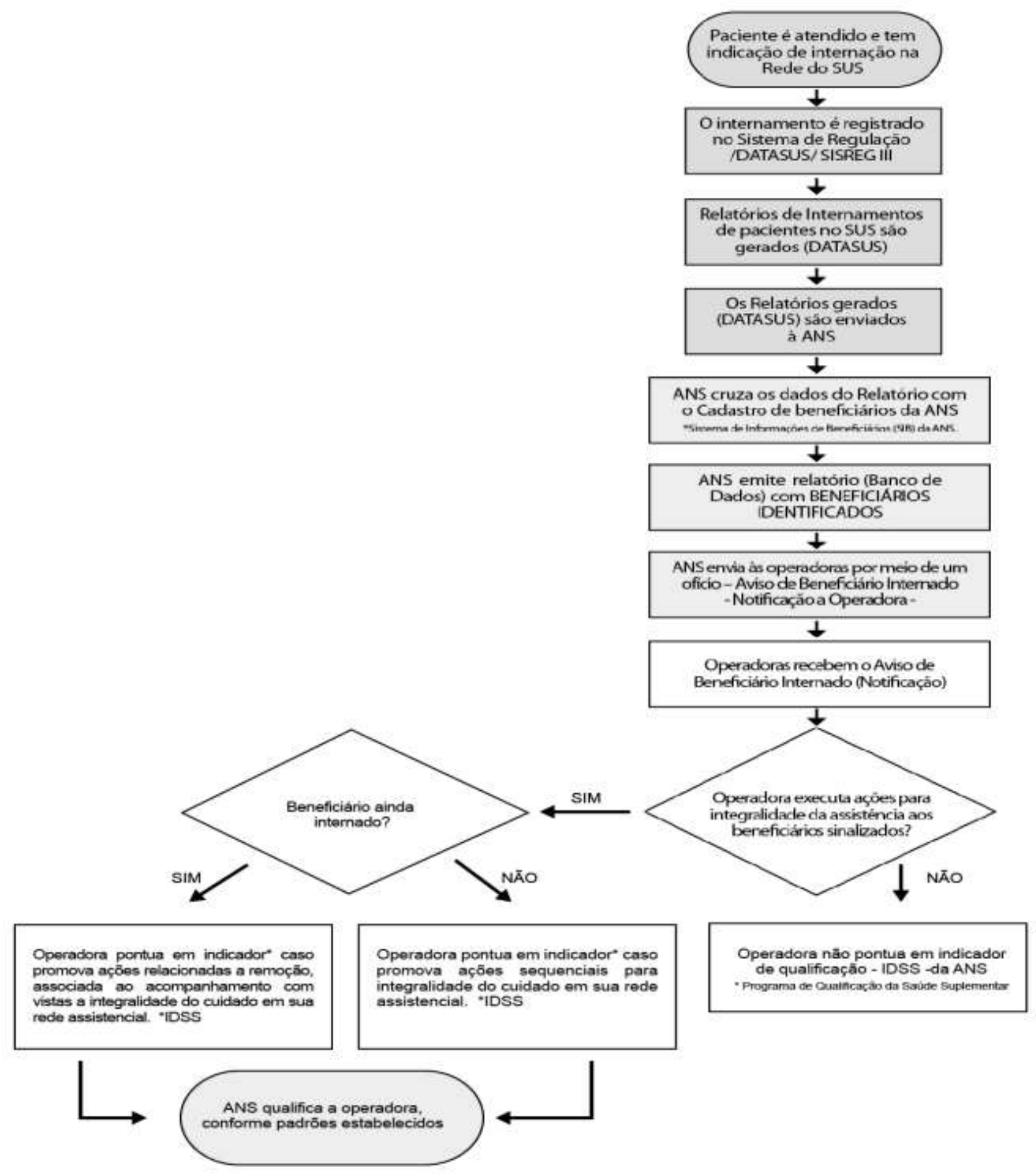

Fonte: Autores.

A versão final do Fluxograma para articulação da Saúde Pública e Saúde Suplementar sob a ótica da integralidade do cuidado traz em sua etapa inicial a indicação da entrada de qualquer indivíduo, com ou sem plano de saúde, ao atendimento que origina um internamento na rede hospitalar do SUS. O atendimento do indivíduo, bem como a indicação para o 
internamento, gera a abertura do processo no Sistema de Regulação/DATASUS/ SISREG III. A depender do município/Estado, podem ser encontrados Sistemas informatizados de regulação com outras denominações que não o SISREG, mas que operam seguindo seus padrões, a exemplo do Unisus, Sistema adotado no Estado do Ceará.

Cada Sistema de Regulação adotado pela localidade (Município/Estado) emite suas informações sobre internamentos através de relatórios específicos. No fluxograma proposto, o relatório de internamentos de pacientes, com a descrição de dados básicos que permitam a identificação dos que foram internados no SUS e que são beneficiários de Planos de Saúde, tais como: Nome do Paciente, Data de Nascimento e Nome da Mãe, é gerado e enviado à ANS. Para esse envio, sugere-se a mesma rotina de envio das informações para o ressarcimento ao SUS, contudo, em prazo inferior, considerando que para se trabalhar a integralidade do cuidado, as operadoras de planos de saúde precisam ser acionadas em tempo hábil para melhor atuação junto aos seus beneficiários.

$\mathrm{Na}$ etapa seguinte propõe que a ANS, ao receber os relatórios, cruze os dados recebidos com o cadastro de beneficiários da ANS ancorado no Sistema de Informações de Beneficiários (SIB) da Agência, como ocorre no fluxo do ressarcimento ao SUS. Sugere-se que o relatório gerado com os "BENEFICIÁRIOS IDENTIFICADOS" seja enviado às operadoras por meio de ofício de Notificação a Operadora de Plano de Saúde, cuja proposta de denominação é “Aviso de Beneficiário Internado".

Ao receberem o Aviso de Beneficiário Internado (Notificação), via ANS, sugere-se que as Operadoras executem ações com vistas à continuidade da integralidade do cuidado, consolidando suas práticas como promotoras de saúde, que é objeto de incentivo da ANS.

Nessa perspectiva, a avaliação de desempenho das operadoras se faz através do Índice de Desempenho da Saúde Suplementar (IDSS), calculado a partir de indicadores definidos pela própria Agência e dentro de 4 (quatro) dimensões, quais sejam: Qualidade em atenção à saúde, Garantia de acesso, Sustentabilidade no mercado e Gestão de processos e regulação (Ministério da Saúde, 2015).

A partir desse momento, o fluxograma subdivide as ações positivas das operadoras após o recebimento da Notificação, distinguindo-as conforme a conduta das mesmas frente a situação em que o beneficiário ainda se encontra internado no SUS, e naquela em que o beneficiário já recebeu alta, quais sejam:

Beneficiário ainda Internado: a operadora pontua caso promova ações relacionadas à remoção para sua rede assistencial, como providenciar a remoção ou comprove a oferta, associada ao acompanhamento com vistas à integralidade do cuidado.

Beneficiário não Internado: a operadora pontua caso comprove ações sequenciais para integralidade do cuidado em sua rede assistencial.

As operadoras que não comprovarem a execução de ações não devem sofrer sanções, entretanto, não se qualificam junto à Agência, o que as expõem negativamente frente ao mercado de Saúde Suplementar, considerando que não demonstram a qualidade de sua atuação e de seus serviços através dos resultados assistenciais que são divulgados à sociedade por meio do site da ANS.

Por ser uma ferramenta propositiva, sem outros fluxos e/ou processos comparativos, os prazos, a disponibilização de notificações, os indicadores, os critérios e os pesos para pontuações, assim como rotinas de acompanhamento e de qualificação das OPS não puderam ser determinados nesse estudo. 


\section{Considerações Finais}

O estudo teve como proposta validar um fluxograma para promover a articulação da Saúde Pública e Suplementar sob a ótica da integralidade do cuidado, considerando que atualmente essa articulação acontece na perspectiva do ressarcimento ao SUS, em âmbito econômico-financeiro. Já a construção do fluxograma teve etapas planejadas e sistematizadas para a garantia de sua operacionalização, execução possível e minimização de equívocos operacionais que o inviabilizassem. As dificuldades enfrentadas estão relacionadas à ausência de fluxos comparativos, e ao distanciamento da saúde pública e saúde suplementar no quesito assistencial e da integralidade do cuidado.

A Validação de Conteúdo e Aparência por juízes especialistas qualificados seguiu o rigor científico, o permitiu a produção de uma ferramenta prática, que coloca a esfera público-privada da saúde em contato e responsabiliza os diversos segmentos dessa rede para a assistência e integralidade do cuidado.

Para que essa ferramenta seja aplicada, faz-se necessário a implementação dos processos atuais. A esfera governamental necessita estudar as etapas propostas, a fim de operacionalizá-las em uma perspectiva proativa com desfecho favorável a ações para integralidade do cuidado.

O Fluxograma Descritor produzido se constitui em proposta inovadora para o Sistema de Saúde, vez que além de propor a articulação para continuidade da integralidade do cuidado na Saúde suplementar, pode contribuir para uma ampliação na oferta de leitos no SUS; a implantação de um processo formal de comunicação, entre os técnicos reguladores do SUS e das Operadoras de saúde; a aproximação da Saúde Pública e da Saúde Suplementar, no que se refere a responsabilidade conjunta na assistência integral aos indivíduos; a redução operacional no número de processos de ressarcimento ao SUS, assim como, incentivar que as operadoras trabalhem iniciativas e estratégias que melhorem gradativamente seus desempenhos assistenciais.

\section{Referências}

Abbagnano, N. (1998). Dicionário de Filosofia. Martins Fontes.

Bardin, L. (2011). Análise de Conteúdo. Edições 70.

Bringer, J. D., Johnston L. H., \& Bracknridge, C. H (2006). Using computer-assisted qualitative data analysis software to develop a grounded theory project. Field Methods. 18(3):245-266.

Corrêa, A. A., Marrero, L., Porto, G. A. C., Silva, D. W. R., Silva, A. C. R. da., Pimenta, I. T., Chaves, J. N., Sousa, O. D., Alves, N. C. M., Brito, R. G. de., \& Pinheiro, F. N. A. (2021) Construção e validação de tecnologia educacional para atenção domiciliar. Pesquisa, Sociedade e Desenvolvimento, 10(3), e40410313532. 10.33448 / rsd-v10i3.13532.

Doak, C., Doak, L., \& Root, J. (1996). Teaching patients with low literacy skills. J. B. Lippincott.

Fehring, R. J. (1994). The Fehring model. In: Carrol-Johnson RM, Paquette M, editores. Classification of nursing diagnoses: proceedings of the tenth conference. Philadelphia: J. B. Lippincott; 55-62.

Franco, T. B. (2003). O Uso do fluxograma descritor e projetos terapêuticos para análise de serviços de saúde, em apoio ao planejamento: o caso de Luz MG. In: Merhy, E. E., Magalhães Junior, H. M., Rimoli, J., et al., organizadores. O Trabalho em Saúde: olhando e experienciando o SUS no cotidiano. São Paulo: HUCITEC. 166-168.

Franco, T. B., \& Merhy, E. E. (2003). O uso de ferramentas analisadoras dos serviços de saúde: o caso do serviço social do hospital das clínicas da UNICAMP. In: Merhy E. E., Magalhães Junior H. M., Rimoli J., et al., organizadores. O Trabalho em Saúde: olhando e experienciando o SUS no cotidiano. São Paulo: HUCITEC. 189-212.

Joaquim, F. L., \& Silvino, Z. R (2020). Validação de tecnologia de gestão para o cuidado de pacientes com úlcera venosa crônica, atendidos em ambulatório em contexto hospitalar. Pesquisa, Sociedade e Desenvolvimento, 9(8), e103985256, 10.33448 / rsd-v9i8.5256.

Likert, R. (1932). A technique for the measurement of attitudes. Arch Psychol.

Lynn, M. R. (1986). Determination and qualification of content validaty. Nurs. Res. 35(6):382-385.

Mesquita, K. S. F. (2016) Fluxograma de apoio à tomada de decisões para assistência de enfermagem à pessoa em sofrimento psíquico na atenção básica [dissertação]. Universidade Federal de Alagoas. $166 \mathrm{f}$.

Ministério da Saúde (1988a). Constituição, 1988. Constituição da República Federativa do Brasil. Senado Federal. 
Research, Society and Development, v. 10, n. 15, e05101522589, 2021

(CC BY 4.0) | ISSN 2525-3409 | DOI: http://dx.doi.org/10.33448/rsd-v10i15.22589

Ministério da Saúde (1988b). Lei nº 9.656, de 3 de junho de 1998. Dispõe sobre os planos e seguros privados de assistência à saúde. Diário Oficial da União.

Ministério da Saúde (2009). Agência Nacional de Saúde Suplementar (Brasil). Atenção à saúde no setor suplementar: evolução e avanços do processo regulatório. ANS.

Ministério da Saúde (2011). Agência Nacional de Saúde Suplementar (Brasil). Manual técnico para promoção da saúde e prevenção de riscos e doenças na saúde. (4a ed.) ANS.

Ministério da Saúde (2015). Agência Nacional de Saúde Suplementar (Brasil) Resolução $n^{\circ}$ 386, de 9 de outubro de 2015. Dispõe sobre o Programa de Qualificação de Operadoras e dá outras providências. Diário Oficial da União.

Nascimento, J., Gomes, I. M., \& Lacerda, M. R. (2016) Uso del software NVivo® en una investigación con Teoría Fundamentada. Index de Enfermería

Pasquali, L. (2013). Psicometria: teoria dos testes na psicologia e na educação. (5a ed.), Vozes.

Polit, D. F., \& Bec, C. T. (2011). Fundamentos de pesquisa em enfermagem: avaliação de evidências para a prática da enfermagem. (7a ed.), Artmed.

Pope, C., \& Mays N. (2009). Pesquisa qualitativa na atenção à saúde. (3a ed.), Artmed.

Portugal, L. B. A., Christovam, B. P., \& Almeida, B. L. O. da S. (2021). Construção e validação da cartilha educativa para enfermeiros sobre lesões por pressão. Pesquisa, Sociedade e Desenvolvimento. 10(3), e3810312926. 10.33448 / rsd-v10i3.12926.

Souza, C. S., Turrini, R. N. T., \& Poveda, V. B (2015) Tradução e adaptação do instrumento "suitability assessmente of materials" (sam) para o português. Rev enferm UFPE on Line

Vianna, H. M. (1982). Testes em educação. IBRASA. 